\title{
The effect of various cyclic Wet-Dry exposure cycles on the Failure Process of Organic Coatings
}

\author{
Shuai Qu ${ }^{1,2}$, Pengfei Ju ${ }^{3}$ Yu Zuo ${ }^{1}$, Xuhui Zhao ${ }^{1 *}$, Yuming Tang ${ }^{1 *}$ \\ ${ }^{1}$ Beijing Key Laboratory of Electrochemical Process and Technology for Materials, Beijing \\ University of Chemical Technology, Beijing 100029, China \\ ${ }^{2}$ AVIC BIAM New Materials Technology \& Engineering Co., Ltd, Beijing 100095, China \\ ${ }^{3}$ Shanghai Aerospace Equipment Manufacture Co. Ltd., Shanghai 200245, China \\ *E-mail: xhzhao@mail.buct.edu.cn, tangym@mail.buct.edu.cn
}

doi: $10.20964 / 2019.12 .52$

Received: 6 August 2019 / Accepted: 20 September 2019 / Published: 29 October 2019

\begin{abstract}
The effect of various dry-wet cycles on the failure processes of the acrylic polyurethane heat-reflection topcoat and thick epoxy anti-rust primer was investigated, where simulated seawater constituted the wet condition and air constituted the dry environment. Electrochemical impedance spectroscopy (EIS), Fourier Transform infrared spectroscopy (FT IR) and Scanning electron microscopy (SEM) were used to characterize the coatings after exposure under the cyclic wet-dry conditions. The results herein indicate that the primary cause of coating degradations was the reaction of functional groups in coatings with the $\mathrm{NaCl}$ solution (the simulated seawater). Furthermore, alternate wetting and drying can promote the incidence of fillers falling off the coatings, and thus, increasing the micro-pores in the coatings. This pore-creating effect can multiply the number of defects in the coating and accelerate the degradation of the coatings. However, the degradation rate of the coatings is evidently influenced by the wet-dry cycle. Under conditions of the same total test times, increased length of the per-cycle wetting times accelerates the coating degradation even though the total wetting times may be equal. If the wetting time in one wetdry cycle is shorter, the water absorption of the coating is lesser. During the following drying process, the absorbed water within the coating may be completely evaporated, and the hydrolysis of the coating can weaken. If the wetting time per cycle is long enough, the absorbing water does not fully evaporate from the coating during the following drying period. This can accelerate degradation of the coating. Moreover, oxygen accelerates the reaction on the cathode during the drying period in one cycle, leading to a relatively quick corrosion of metal matrix and a decrease of adhesive force of the coating.
\end{abstract}

Keywords: acrylic polyurethane heat-reflection coating, thick epoxy antirust coating, wet-dry cycle, Failure, EIS.

\section{FULL TEXT}


(C) 2019 The Authors. Published by ESG (www.electrochemsci.org). This article is an open access article distributed under the terms and conditions of the Creative Commons Attribution license (http://creativecommons.org/licenses/by/4.0/). 